Int. J. Dev. Biol. 56: 799-808 (2012)

doi: $10.1387 / \mathrm{ijdb} .120140 \mathrm{ec}$

\title{
Metabolism throughout follicle and oocyte development in mammals
}

\author{
ESTHER COLLADO-FERNANDEZ ${ }^{*, 1}$, HELEN M. PICTON ${ }^{1}$ and RÉMI DUMOLLARD, ${ }^{* 2}$ \\ ${ }^{1}$ Division of Reproduction and Early Development, Leeds Institute of Genetics, Health and Therapeutics, University of \\ Leeds, UK and ${ }^{2}$ Developmental Biology Unit UMR 7009, UMPC Univ. Paris 06 and Centre National de la Recherche
} (CNRS), Observatoire Océanologique, France

\begin{abstract}
Metabolic studies of mammalian embryos started with the development of in vitro culture systems more than $\mathbf{4 0}$ years ago. More recently, metabolic studies have begun to shed light on the requirements of growing oocytes/follicles from the earliest stages of folliculogenesis. While growing oocytes preferentially metabolise pyruvate over glucose, the somatic compartment of ovarian follicles is more glycolytic. The metabolic preferences of the oocyte are reflected in the early zygote, which becomes increasingly dependent on glycolytic energy production as development progresses to the blastocyst stage. Furthermore, the intricate metabolic relationship between each oocyte and its somatic surroundings is critical for oocyte growth and developmental competence. Measurements of amino acid turnover in bovine oocytes indicate that glutamine, arginine and leucine are consistently depleted, while alanine is produced, showing similarities with amino acid turnover in preimplantation embryos. Amino acid profiling is a good predictor of embryo quality and might also turn out to be a predictor of oocyte developmental competence. Finally, recent studies have uncovered lipid metabolism in oocytes and early embryos, suggesting that endogenous fatty acids might be used for energy production. Together, metabolic studies have revealed the multiplicity of energetic substrates used by oocytes and early embryos, and suggest that the versatility of the metabolic pathways available for energy production is key for high developmental potential. Metabolic studies of early embryos are now being applied to follicle culture, and the goal of describing the metabolome of the growing oocyte in its follicle is now very attainable.
\end{abstract}

KEY WORDS: oocyte, follicle, metabolism, carbohydrate, amino acid, lipid

\section{Introduction}

The revolution of metabolomics is promising to deliver an exhaustive description of the oocyte's metabolome, and to reveal the metabolome of a healthy oocyte in order to discriminate (non invasively) which gamete has the greatest chance of producing a successful pregnancy. Numerous studies have described the metabolic pathways operating in oocytes and follicles. Metabolomic studies must also describe the metabolite fluxes in the oocyte in order to decipher the respective contribution of each metabolic pathway during oocyte development. The abundance of biochemical studies published since the development of in vitro culture of mammalian embryos in the 1960s, provides the opportunity to tentatively start describing the oocyte metabolome to possibly direct future studies employing modern techniques. Metabolic studies -which initially used radiolabelled substrates-made it possible to define the metabolites that mammalian oocytes and embryos require. More recently, amino acid analysis of spent culture media has revealed the usage of different amino acids by oocytes (Hemmings et al., 2012) and embryos (Sturmey et al., 2008) in a range of species,

\footnotetext{
Abbreviations used in this paper: AMP, adenosine monophosphate; ATP, adenosine triphosphate; $\mathrm{CC}$, cumulus cell; COC, cumulus-oocyte complex; CPT1, carnitine palmitoyl transferase 1; Cx, connexin; EGA, embryo genome activation; EGF, epidermal growth factor; G6PDH, glucose-6-phosphate dehydrogenase; GC, granulosa cells; GSH, glutathione; GV, germinal vesicle; GVBD, germinal vesicle breakdown; HBP, hexosamine byosinthetic pathway; hCG, human chorionic gonadotropin; HPLC, high performance liquid chromatography; IVM, in vitro maturation; LDH, lactate dehydrogenase; LH, lutheinising hormone; MII, metaphase II; NADH, nicotinamide adenine dinucleotide (reduced form); NADPH, nicotine adenine dinucleotide phosphate (reduced form); Oxphos, oxidative phosphorylation; PGG, primordial germ cell; PPP, pentose phosphate pathway; TCA cycle, tricarboxilic acid cycle.
}

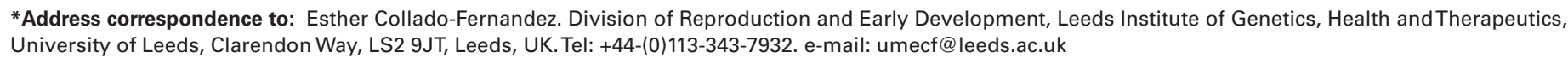


and is providing insights into the amino acids utilized by healthy oocytes (Hemmings et al., 2012). Indeed, in pre-implantation embryos the quantification of amino acid and energy metabolism is emerging as a potential non-invasive tool with which to assess embryo developmental competence and even pregnancy outcome (Sturmey et al., 2008; Picton et al., 2010; Gardner et al., 2011).

Mammalian follicle and oocyte metabolism has been studied mainly in antral follicles and fully grown oocytes. Little is known about follicle/oocyte metabolism at earlier stages of folliculogenesis. Studies in murine follicles and oocytes, suggest that their metabolic profiles change dynamically as follicle development progresses (Harris et al., 2007; Harris et al., 2009). A better understanding of the metabolism of early follicles and oocytes is important as they represent the greatest proportion of follicles in the female ovaries. Its study is of value for the development of better culture systems for in vitro growth and in vitro production of oocytes, techniques applied in fertility preservation and stem cell production. In this review we aim to provide an overview of the oocyte and follicle metabolism throughout oogenesis. Metabolism of early embryos will also be mentioned, but we refer the interested reader to reviews on embryo metabolism (Johnson et al., 2003; Dumollard et al., 2009; Leese, 2012). After briefly describing oogenesis and folliculogenesis, we review studies addressing carbohydrate and amino acid metabolism in follicles and oocytes to emphasise how the metabolic cooperativity between the oocyte and supporting cells underlie such a complex and changing metabolism. Finally, the possibility that endogenous fatty acids might support oocyte development will be discussed.

\section{Oogenesis and folliculogenesis: an overview}

Oocyte development begins in the female mammalian foetus with the differentiation of primordial germ cells (PGC). Proliferating PGC migrate towards the nascent genital ridges, where they differentiate into oogonia, before entering meiosis to become primary oocytes (McLaughlin and Mclver, 2009). During post natal life, oocytes undergo growth and maturation, stockpiling mRNAs, proteins, metabolic substrates and organelles. Such maternal stores must support fertilization, unpacking of paternal genome, karyogamy and early cleavage division of the zygote until embryo genome activation (EGA) begins. Primary oocytes, which remain arrested at diplotene of the $1^{\text {st }}$ meiotic prophase, become enveloped by a single layer of flattened pre-granulosa cells and a basement membrane to form primordial follicles (Fig. 1). The ovarian population of primordial follicles are continuously depleted by atresia or stimulated to grow during follicle activation (McLaughlin and Mclver, 2009). Following growth activation, primordial follicles become primary follicles, which are characterised by a full layer of proliferative cuboidal granulosa cells (GC) (Picton, 2001) (Fig. 1). Over the lengthy process of follicular growth, GC continue to proliferate, the theca layer develops which provides the follicle with an independent blood supply (Young and McNeilly, 2010), and follicles pass through the secondary, preantral and antral stages before final maturation and ovulation (Binelli and Murphy, 2010) (Fig. 1). The antral cavity forms when follicles reach a diameter between 200-500 $\mathrm{um}$ depending on the species (Picton et al., 1998). It becomes filled with follicular fluid, which acts as a source of oxygen, buffering molecules, carbohydrates, amino acids, growth factors, hormones and other molecules (Sutton et al., 2003b). In addition, the formation of the antrum leads to the differentiation of GC into two spatially and functionally distinct populations: the mural granulosa cells, which line the basement membrane and are characterised by their endocrine role; and the cumulus cells, which are intimately associated with the oocyte and support its metabolism and maturation. Both cell types form the cumulus-oocyte complex (COC) (Fig. 1).

Within the growing follicle, the oocyte undergoes a 100-300-fold increase in volume (Griffin et al., 2006), which is largely completed around the time the antrum is formed, and is supported by an increasing number of surrounding GC (Griffin et al., 2006) (Fig. 1 ). The ooplasm of the growing oocyte accumulates glycogen granules, lipid droplets -especially in farm animals (Sturmey et al., 2009)-, proteins and mRNA. The endoplasmic reticulum and Golgi complexes are abundant and undergo structural changes and redistribution, reflecting the high synthetic activity of the oocyte (Fair et al., 1997; Picton et al., 1998). The number of vesicles and ribosomes increases, cortical granules appear and glycoproteins are exported to form the zona pellucida (Fair et al., 1997; Picton et al., 1998). Glutathione (GSH), a glutamate-cysteine-glycine tripeptide that protects the oocyte from oxidative stress and is re-

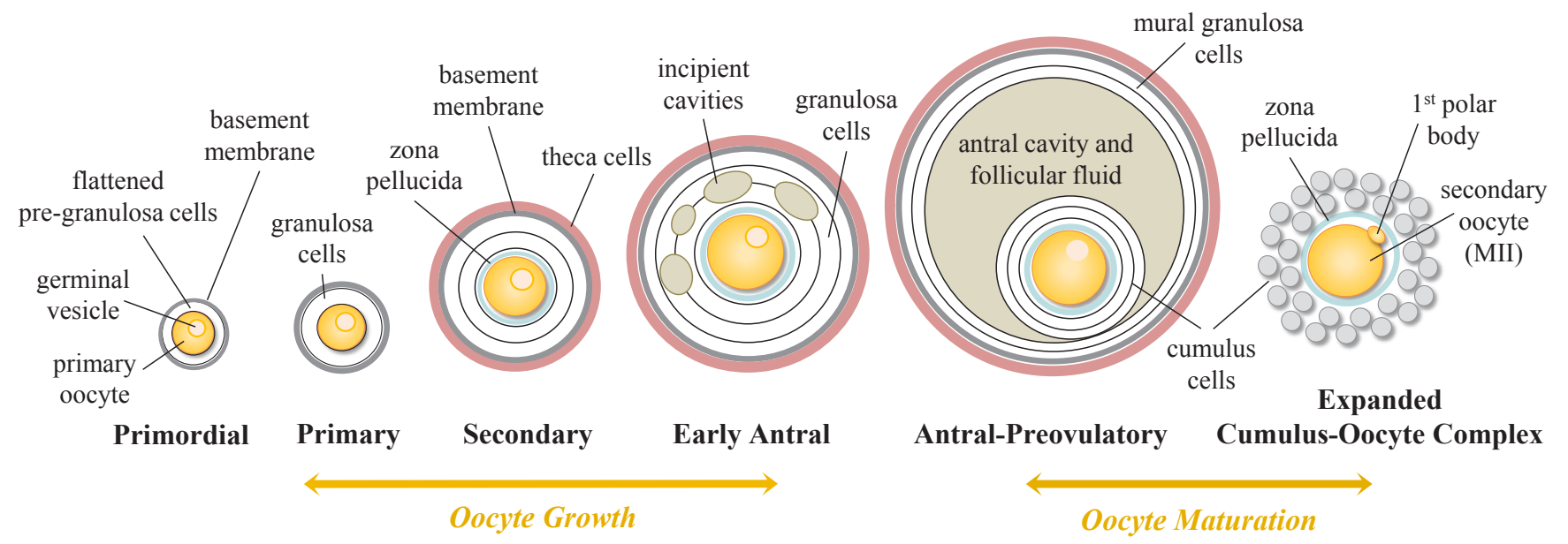

Fig. 1. Simplified representation of mammalian folliculogenesis and oocyte growth. Follicle developmental stages are indicated, and the different cell layers at each stage are represented as full lines without cellular divisions. 
quired for the decondensation of the sperm nucleus and formation of the male pronucleus, also accumulates during oocyte growth and maturation (reviewed by Luberda (2005)). Mitochondria also replicate during oogenesis, and their number rises from about 200 per oogonia to 6,000 per oocyte in primordial follicles, and reaches up to $300,000-400,000$ in the mature human oocyte (Jansen and de Boer, 1998). It is hypothesised that such a rise in mitochondria number compensates for their apparent lack of maturity and activity. In turn, the low activity of individual mitochondria is thought to minimise oxidative stress (Dumollard et al., 2006). Mitochondrial replication then ceases, and resumes only after implantation (reviewed by Jansen and de Boer (1998) and Dumollard et al.(2006)). The expansion of the mitochondrial population during oogenesis is essential for supplying all the mitochondria of the preimplantation embryo, and as such it is vital for preimplantation development.

Selection and final growth of the follicle/s destined to ovulate is mediated by the preovulatory surge of gonadotropins, which triggers COC expansion, gap junction loss, and ultimately oocyte maturation and ovulation (Binelli and Murphy, 2010). Oocyte maturation is comprised of both, nuclear and cytoplasmic maturation, and both must be completed for the gamete to acquire developmental competence (Eppig, 1996). Nuclear maturation consists of the nuclear changes associated with the resumption of meiosis I (germinal vesicle breakdown (GVBD), progression to metaphase II (MII) and extrusion of the first polar body, and arrest at MII (for a review see (Tripathi et al., (2010)). In contrast, cytoplasmic maturation comprises the acquisition of mechanisms for sperm penetration and polyspermy block, calcium release and exocytosis of cortical granules, decondensation of the sperm chromatin, pronuclei formation, and support of the first embryonic divisions (Ajduk et al., 2008; Ferreira et al., 2009). Post-transcriptional modification of stable mRNAs stored during oocyte growth, protein synthesis and post-translational regulation are also a critical part of cytoplasmic maturation (Picton et al., 1998). In addition, oocyte mitochondria redistribute during GVBD and progression to MII, and altered mitochondrial redistribution is associated with lower oocyte developmental potential. The multiple and pervasive functions of mitochondria in oocytes and early embryos have been reviewed recently and will not be discussed further (Dumollard et al., 2006; Dumollard et al., 2007; Dumollard et al., 2009; Van Blerkom, 2011).

Oocyte developmental competence has been shown to be acquired progressively during oocyte growth in different mammalian species (for a review see (Trounson et al., 2001). Many of the dynamic processes occurring during oocyte growth and maturation require energy, and oocytes and follicles can exploit a variety of metabolites to support their energetic and anabolic needs.

\section{Carbohydrate metabolism throughout folliculogenesis}

Energy metabolism of PGC and oogonia was suggested to be mainly anaerobic and glycolytic, thus avoiding oxidative damage (Jansen and de Boer, 1998). PGCs contain a large nucleus, few organelles, abundant glycogen and occasional lipid droplets, which could provide a source of energy during their migration (Motta et al., 1997). However, PGCs have also been shown to preferentially oxidise pyruvate over glucose (Brinster and Harstad, 1977 ) indicating aerobic oxidation in PGCs. Besides, primordial follicles readily produce lactate, and consume 2 -fold more pyruvate than glucose, indicating that both glycolysis and mitochondrial pyruvate oxidation operate at this stage, and that pyruvate might be the main energy substrate (Harris et al., 2009). Oocytes at the earliest stages of development are not quiescent. Indeed, when the substrate preference by oocytes of a diameter approximately corresponding to those within primordial follicles was studied, no oxidation of glucose to $\mathrm{CO}_{2}$ was detected, only pyruvate oxidation was observed (Eppig, 1976); and at a rate similar to that of primordial follicles as described by Harris et al., (2009). Thus, it appears that the small number of pre GC in primordial follicles could be responsible for the little glucose consumption and lactate production by the intact follicle, while pyruvate is consumed by denuded primordial oocytes (Harris et al., 2009). Species-specific differences in substrate preferences might nevertheless exist, as shown for fully grown oocytes (Krisher et al., 2007).

With the initiation of follicle and oocyte growth, a highly active period starts. Unlike resting primordial follicles, which are located in the poorly vascularised outer ovarian cortex, growing follicles are found in the well vascularised cortico-medullary border (Picton, 2001). In addition, the energetic demands of the growing oocyte seem to be reflected in the increasing pyruvate and oxygen consumption by murine oocytes throughout the growth period (Harris et al., 2009). Indeed, when data was corrected for oocyte volume, oocytes from primary follicles showed a particularly high metabolic turnover (Harris et al., 2009) (Fig. 2 B,C). Glucose uptake was not detected at any stage of murine oocyte growth (Harris et al., 2009), indicating a low glycolytic activity. This is in agreement with studies in fully grown oocytes, where although glucose consumption and metabolism by denuded oocytes was detected, glucose represented a minor energetic substrate (Rieger and Loskutoff, 1994; Sutton-McDowall et al., 2010). Follicle glucose consumption and lactate production rates increase as murine follicles develop in vitro from primary/secondary stages to antral and ovulatory-like stages (Boland et al., 1994a; Harris et al., 2007) (Fig. 2A). Nevertheless, the low ratio of glucose consumption to lactate production suggests that glycolysis contributes poorly to energy production during early preantral stages (Harris et al., 2007). This pattern appears to change around the time of antrum formation, with antral follicles being regarded as predominantly glycolytic. Such increased glycolytic activity might be related to low oxygen availability, initiation of oestrogen synthesis and increasing energy demands (Boland et al., 1993; Boland et al., 1994b; Harris et al., 2007).

When the growing follicle reaches a size at which supply from theca capillaries could be compromised, because of the thickening and nutritional requirements of the GC layer, the follicular fluid acts as a source of oxygen and nutrients (Redding et al., 2007). Mathematical modeling was used to study the mean oxygen concentration in human follicles; and predicted a low oxygen concentration within large preantral follicles, yet sufficient to reach the oocyte (Redding et al., 2007). Other studies in the human (Redding et al., 2008) and bovine (Clark and Stokes, 2011) predict a sharp rise in intrafollicular oxygen with antrum formation, peaking at the end of the early antral stage before decreasing through the antral to preovulatory stages, and to finally increase around the time of ovulation. Predicted and actual measurements of oxygen concentration in antral follicles are similar to those in venous blood (Redding et al., 2008). Thus, the oxygen supply to the oocyte may only be limited at the late preantral to early antral stage and at the early preovulatory stage (Redding et al., 2007, 2008). Follicle angiogenesis at these stages could be crucial for oocyte oxygen- 
ation and development (Van Blerkom, 2000).

The final stages of folliculogenesis in vivo are marked by the preovulatory surge of gonadotropins. In vitro, gonadotropins cause a dramatic increase in follicle glucose uptake and lactate production (Roberts et al., 2004; Sutton-McDowall et al., 2004; Harris et al., 2007) (Fig. 2A). Such actions are likely mediated by up-regulation of glucose transporters (Kol et al., 1997; Roberts et al., 2004) and glycolytic enzymes (Downs et al., 1996; Roy and Terada, 1999). Glucose metabolism is also influenced by insulin and ovarian growth factors (Roy and Terada, 1999). Yet, the proportion of glucose metabolised by glycolysis has been shown to diminish following follicle/COC maturation, suggesting that some glucose is metabolised through an alternative pathway/s (Sutton et al., 2003a; Sutton-McDowall et al., 2004; Harris et al., 2007). One of these pathways is probably the PPP (Downs et al., 1998). This is supported by the relative high activity of the PPP rate limiting enzyme glucose-6-phosphate dehydrogenase (G6PDH) compared to the glycolytic enzyme phosphofructokinase in bovine oocytes during in vitro maturation (IVM) (Cetica et al., 2002). Yet, a decrease in G6PDH activity in fully grown oocytes has been reported, and suggested to be linked to oocyte cytoplasmic maturation (Su et al., 2012; Ishizaki et al., 2009). Furthermore, it is well established that glucose is required to support $\mathrm{FSH}$-dependent cumulus expansion and oocyte maturation (Fagbohun and Downs, 1992), while denuded mouse oocytes require pyruvate or oxaloacetate to sustain spontaneous nuclear maturation (Biggers et al., 1967; Downs and Hudson, 2000). The oocyte energetic requirements for resumption of meiosis appear to be met by an increase in oxidative metabolism around the time of GVBD; pyruvate being used as the main substrate, and supplemented by glutamine and glycine which can feed into the TCA cycle (Zuelke and Brackett, 1993; Rieger and Loskutoff, 1994; Downs et al., 2002; Harris et al., 2007). Metabolites such as glucose and fatty acids are also thought to play a key role in bovine and porcine oocyte maturation (Krisher et al., 2007; Sturmey et al., 2009).

Within the follicle glucose can be metabolised through: (i) glycolysis -producing adenosine triphosphate (ATP) and pyruvate or lactate; (ii) the pentose phosphate pathway (PPP) -providing precursors of purine nucleotides and nicotine adenine dinucleotide phosphate hydrogen (NADPH) for biosynthetic pathways and antioxidant defence. Although metabolic flux though the PPP might be low, it is thought to be key for COC maturation; (iii) the hexosamine biosynthetic pathway (HBP) -by which glucose and glutamine are involved in protein glycosylation as well as hyaluronic acid synthesis for cumulus expansion; and (iv) the polyol pathway producing sorbitol and fructose -whose role remains largely unknown (for a review see (Sutton-McDowall et al., 2010)). Glucose-derived or extracellular pyruvate can be metabolised in the mitochondria through the tricarboxylic acid (TCA) cycle and oxidative phosphorylation (oxphos), which produces most of the ATP in the oocyte (Dumollard et al., 2007; Dumollard et al., 2009). Lactate, highly abundant in follicular fluid and the reproductive tract (Harris et al., 2005), is oxidised to pyruvate by cytosolic lactate dehydrogenase (LDH). Interestingly, such lactate-derived pyruvate does not fuel mitochondrial ATP production in denuded mouse oocytes, instead lactate is important for regulation of the cytosolic redox state (Dumollard etal., 2007; Dumollard et al., 2009). Mitochondria also metabolise ketone bodies originating from deamination of amino acids such as leucine, and are responsible for the catabolism of fatty acids through $\beta$-oxidation. While GC are mainly
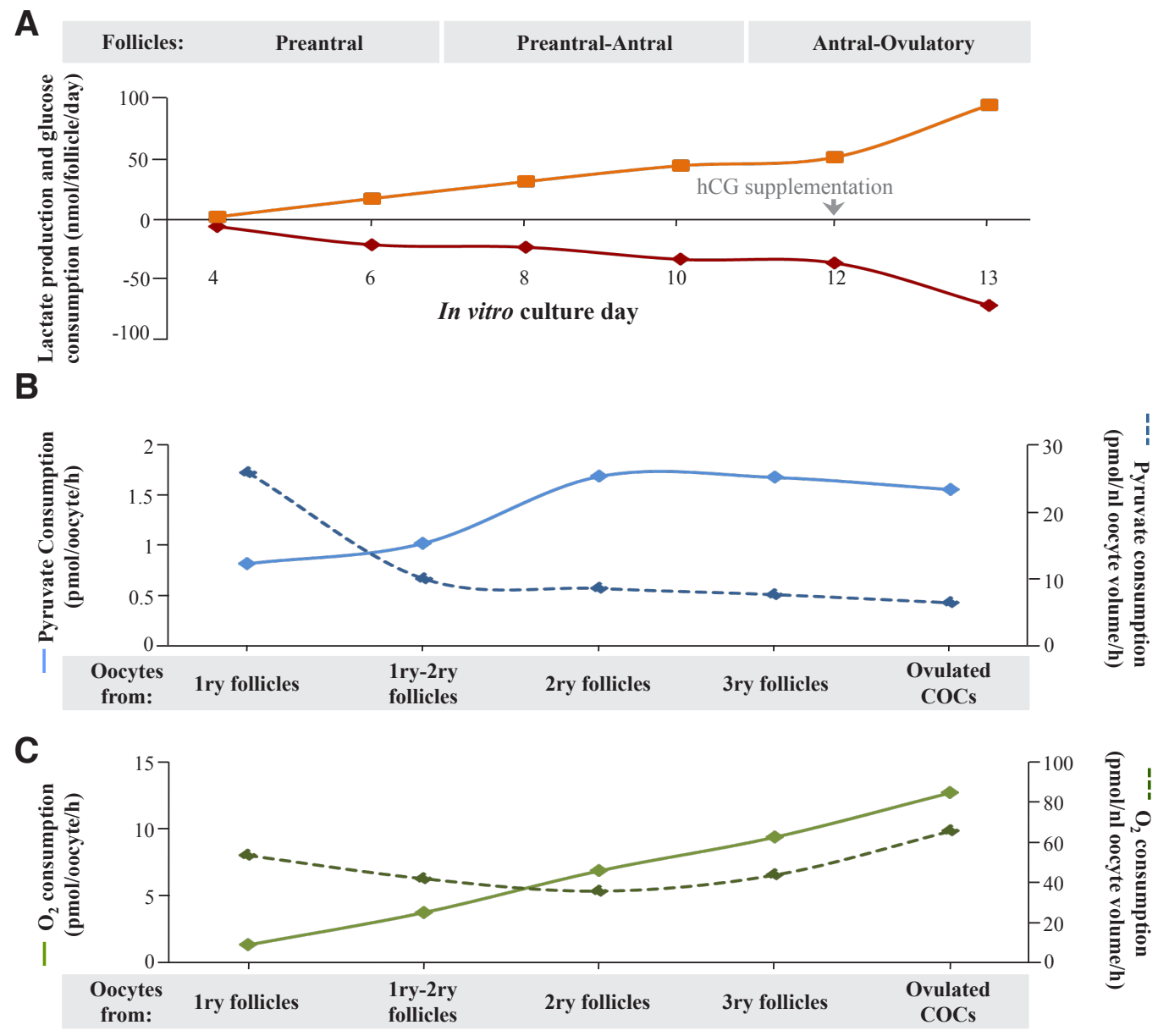

Fig. 2. Carbohydrate metabolism of murine follicles and oocytes throughout development. (A) Lactate production (positive values) and glucose consumption (negative values) by in vitro grown follicles, which were at the late primary to secondary stage at the beginning of culture. Follicle developmental stages and culture days are indicated (adapted from Harris et al., 2007). (B) Pyruvate consumption and (C) oxygen consumption by oocytes from follicles at different stages. Follicle stages are indicated. Dashed lines indicate data corrected for oocyte volume (adapted from Harris et al., 2009). 
glycolytic, oocyte energy metabolism relies mainly on mitochondrial oxidation of extracellular pyruvate as well as metabolites supplied by the somatic compartment. Mitochondrial oxidative metabolism is the major oxygen consumer in the follicle and the oocyte, and -besides mitochondrial oxphos- oxygen is required for synthesis of cholesterol and steroidogenesis in GC of antral follicles (Boland et al., 1994a), and for $\beta$-oxidation of intracellular lipid stores (see below). The requirement for oxygen is illustrated by the detrimental effect anoxic culture conditions have upon murine follicle development and steroidogenesis (Boland et al., 1994a). Disruption of the TCA cycle in murine oocytes resulted in compromised nuclear maturation and developmental competence (Johnson et al., 2007). There is now strong evidence that mitochondrial function supports ATP production and $\mathrm{Ca}^{2+}$ homeostasis, and sets the cellular redox state in the mouse oocyte and embryo; and such functions are key to the oocyte and embryo developmental competence (for a review see (Johnson et al., 2003; Dumollard et al., 2007; Dumollard et al., 2009; Van Blerkom, 2011).

Cumulus expansion represents the end of the oocyte-CC metabolic cooperativity. The oocyte and preimplantation embryo then rely on internal reserves and the external medium for provision of metabolites. It is important to bear in mind the changing environment from that within the follicular fluid, through that in the post ovulatory oviduct fluid and later in the uterus (Fischer and Bavister, 1993; Harris et al., 2005). There is no specific blood supply following ovulation and until well after implantation (Burton et al., 2010), and significant variations in oxygen, carbohydrate and amino acid concentration along the reproductive tract have been reported (Fischer and Bavister, 1993; Harris et al., 2005; Hugentobler et al., 2007).

Changes in (i) the environment characteristics, with both restrictive and facilitative action (e.g. oxygen concentration and nutrient supply); and (ii) the increasing embryo requirements for energy and metabolites to support cell division, EGA and blastocoel formation, are reflected in the major changes in embryo metabolism during the preimplantation period. Namely increasing metabolic activity and gradual shift towards greater importance of glucose metabolism (for a review see (Johnson et al., 2003) and (Leese, 2012)). A part from this general pattern, there are important species-specific differences in the use of metabolic substrates and the relative importance of pyruvate and glucose metabolism (Swain et al., 2002).

\section{Amino acid metabolism supports oocyte growth and cytoplasmic maturation}

Amino acids serve as substrates for the synthesis of proteins, nucleotides (glutamine, aspartate, glycine), GSH (glutamate, cysteine, glycine), glycoproteins, hyaluronic acid (glutamine bound to glucose through the HBP), and signalling molecules such as nitric oxide (arginine). They also play important roles as energy substrates (glutamine, glycine, and others), $\mathrm{pH}$ and osmolarity regulators (glycine, alanine, glutamine, and others), heavy metal chelators (glycine), donors of methyl groups (methionine), in the secretion of ammonia (alanine) and in anaplerosis (aspartate, glutamate, leucine, lysine, isoleucine and others) (Dumollard et al., 2007; Sturmey et al., 2008).

Growing oocytes are characterized by a high RNA and protein synthetic activity (Picton et al., 1998). Protein synthesis is also particularly high during the early stages of oocyte maturation (Tomek et al., 2002). For example, the RNA content and the absolute rate of protein synthesis increase 300-fold and 38-fold respectively, during the growth phase of murine oocytes (Schultz et al., 1979; Sternlicht and Schultz, 1981). Glutamine, aspartate, glycine and ribose-5-phosphate (product of glucose metabolism through the PPP) are required for de novo synthesis of purine and pyrimidine nucleotides for mRNA synthesis. A part from protein and mRNA synthesis, some amino acids can also serve other purposes in the oocyte. Indeed, part of the ATP demands could be met by oxidative metabolism of glutamine and glycine through the TCA cycle (Zuelke and Brackett, 1993; Rieger and Loskutoff, 1994).

In comparison to carbohydrate metabolism, and despite their important roles, there is little information about amino acid uptake and metabolism by follicles and oocytes, especially at the earlier stages of folliculogenesis. Studies measuring amino acids in follicular fluid and the reproductive tract provided valuable information about the available substrates, showing significant levels of amino acids. For example glutamine, glycine and alanine are consistently among the amino acids at highest concentrations throughout the reproductive tract in different species (Harris et al., 2007; Hugentobler et al., 2007). Studies investigating amino acid metabolism have mainly focused on the embryo (Van Winkle, 2001; Sturmey et al., 2008), while less information is available from oocytes (Pelland et al., 2009; Hemmings et al., 2012). In addition, although about 18 amino acid-selective transport systems are thought to operate in mammalian embryos, indicating their capacity for amino acid uptake (Van Winkle, 2001), only a few have been identified during oocyte growth and maturation (Pelland et al., 2009). Overall, embryos appear to consume glutamine and arginine and produce alanine consistently during preimplantation development, yet a more specific description of amino acid utilisation depends upon the culture conditions, the developmental stage and the species studied (see for example pig: (Booth etal., 2005); bovine: (Partridge and Leese, 1996); human:(Houghton et al., 2003)).

In the bovine, LH has been shown to increase glutamine oxidative metabolism by oocytes and COCs (Zuelke and Brackett, 1993). Addition of glutamine to bovine IVM media also appears to promote oocyte nuclear maturation (Bilodeau-Goeseels, 2006),

\section{TABLE 1}

\section{AMINO ACID TRANSPORT AND TURNOVER IN GROWING AND FULLY GROWN OOCYTES}

Growing Oocytes

Increasing saturable transport of Ala, Lys, Leu, Gln, Arg ${ }^{(1)}$

Uptake of Ala, Lys, Gly and Tau is enhanced by follicle cells (Ala, Lys and Tau uptake by fully grown oocytes also enhanced by CC) ${ }^{(1,2)}$

Leu uptake is inhibited by the presence of follicular cells ${ }^{(1)}$
Oocytes Undergoing Maturation

Peak in saturable transport of Gly, Tau, cystine

Consumption of Gln and Arg, and production of Ala and Gly by mature bovine oocytes ${ }^{(3)}$

Supplementation of IVM media with some amino acids appears to have beneficial effects upon oocyte maturation and developmental potential in different species (i.e. Gln, Cys) ${ }^{(4)}$

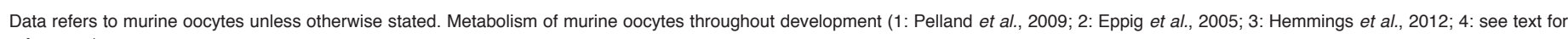
references) 
while supplementation of defined IVM media with non-essential and essential amino acids increases oocyte maternal mRNA levels and enhances embryo development (Watson et al., 2000). In the pig, defined IVM media supplemented with glutamine, aspartate and valine enhanced the rate of monospermic fertilization; while most of the amino acids studied improved male pronucleus formation, arginine and alanine had a beneficial effect on embryo development (Hong and Lee, 2007). In the mouse, the use of glutamine as a sole energy substrate was shown to be sufficient to initiate GVBD, but not to reach MII (Downs and Hudson, 2000), which could be related to an insufficient activity of the PPP (Sutton et al., 2003b).

Three recent studies have provided new information regarding the amino acid turnover of oocytes and follicles over growth and maturation (see Table 1). First, Chand and Legge (2011) measured the uptake of radiolabelled leucine in mouse follicles and reported an increase in the rate of leucine uptake as follicles developed from pre-antral to late antral stages in vitro. A reduction in transport was observed as follicles attained the pre-ovulatory stages (Chand and Legge, 2011). Second, Pelland et al., (2009) characterised the transport systems for nine amino acids (aspartate, glutamine, glycine, arginine, alanine, leucine, lysine, cystine, and taurine) through growth and maturation of murine oocytes. Leucine, arginine and lysine were transported at the highest rates (Pelland et al., 2009). Three different patterns were identified: (i) Aspartate did not show saturable transport and was transported at a very low rate, especially after GVBD. (ii) Saturable transport for alanine, lysine, leucine, glutamine and arginine increased during oocyte growth, and reached a plateau or decreased from $\mathrm{MI}$ to $\mathrm{MII}$, suggesting a role during the energy demanding and stockpiling process of oocyte growth, cytoplasmic maturation and GVBD. (iii) Saturable transport for glycine, cystine and taurine was very low or absent during growth and peaked in $\mathrm{MI}$ and MII oocytes (Pelland et al., 2009). Such patterns of amino acid transport may reflect the demand for glycine and cystine for the major GSH synthesis occurring during oocyte maturation (Luberda, 2005; Pelland et al., 2009). Moreover, six different amino acid transport systems were identified both in growing and mature oocytes. And follicular cells were shown to enhance the uptake of glycine, alanine, lysine and taurine by oocytes (Pelland et al., 2009). Finally, Hemmings et al., (2012) quantified the amino acid profile (i.e. depletion and appearance rates) by bovine MII oocytes after IVM. Glutamine, arginine and asparagine were depleted at the highest rates, while alanine and glycine were released into the media. Similar to studies carried out in embryos, oocytes with higher developmental potential after IVF and those of poorer quality presented different amino acid profiles (Hemmings et al., 2012). Overall, poorer quality was related to higher amino acid turnover, in agreement with Leese's "quiet embryo hypothesis" (Leese et al., 2008). For example, oocytes that failed to cleave depleted more glutamine, released more alanine and presented higher total amino acid depletion, appearance and turnover than oocytes able to cleave (Hemmings et al., 2012). Furthermore, these data was used to predict fertilisation and cleavage potential (Hemmings et al., 2012). These studies provide further evidence of the importance of amino acid metabolism for oocyte developmental competence.

\section{Metabolic cooperativity between the oocyte and granu- losa cell}

The co-ordinated bidirectional communication between the devel- oping follicle and its oocyte is required for the correct development of both compartments. This communication is mediated by extracellular factors and gap junctional coupling. Somatic cells support oocyte development by providing metabolites, and by regulating oocyte growth and maturation (Binelli and Murphy, 2010; Sutton-McDowall et al., 2010). CC are also thought to aid fertilisation (Tanghe et al., 2002). Thus, disruption of CC-oocyte communication before oocyte maturation or fertilisation decreases oocyte developmental competence (Zhang et al., 1995). In turn, oocyte derived factors regulate GC/CC metabolism (Su et al., 2009), theca and GC proliferation and differentiation, as well as cumulus expansion (Binelli and Murphy, 2010).

Homologous gap junctions between GC, and heterologous gap junctions between GC and the oocyte mediate their metabolic coupling by allowing the free transit of small molecules $(<1 \mathrm{kDa})$, including ions, amino acids, pyruvate, glucose, nucleotides and other signalling molecules (Su et al., 2009; Wang et al., 2012). Heterologous gap junctions have been described from the primordial follicle stage in mice (Mitchell and Burghardt, 1986) or the later secondary stage in cattle (Fair et al., 1997). Their importance is evident from the fact that mice deficient for connexin 37 (the major connexin in heterologous gap junctions) are infertile and show follicles arrested at the late preantral stage, premature GC differentiation into luteal cells, and impairment of oocyte growth and meiotic competence (Carabatsos et al., 2000). Similarly, mutation of the gene encoding for connexin 43 (the major connexin in homologous gap junctions) causes follicle arrest at the primary stage, as well as impairment of oocyte growth and meiotic competence (Ackert et al., 2001). These studies seem to indicate that heterologous gap junctions are not essential for early follicle development, yet they appear key for normal oocyte growth and maturation.

Glucose is the preferred energy substrate for CC (Sutton-McDowall et al., 2010), whereas oocytes of most mammalian species studied consume little glucose, pyruvate being the preferred energy substrate (Eppig, 1976; Rieger and Loskutoff, 1994). Glucose appears to be a more important substrate for oocytes of species such as pig and Rhessus monkey (Krisher et al., 2007; Zheng et al., 2007). In general, oocytes take up pyruvate efficiently, but have lower capacity for glucose transport, as well as limited expression and activity of some glycolytic enzymes (reviewed by (Purcell and Moley, 2009; Sutton-McDowall et al., 2010)). Thus, oocytes rely on CC-derived oxidable substrates for synthesis of ATP, namely pyruvate (Biggers et al., 1967; Johnson et al., 2007; Harris et al., 2009). ATP and glucose may also be directly transferred from GC/CC through gap junctions (Downs, 1995; Wang et al., 2012).

Denuded oocytes also have a reduced capacity to take up some amino acids such as alanine, glycine, lysine and histidine. The uptake of such amino acids is enhanced by CC, which appear to pass them into the oocyte via gap junctions (Eppig et al., 2005; Pelland et al., 2009). Conversely, leucine uptake by murine oocytes appears inhibited by the presence of CC (Pelland et al., 2009) (see table 1). When compared to bovine oocytes, CC also possess greater activity of the enzymes involved in amino acid metabolism such as aspartate aminotrasferase and particularly alanine aminotrasferase, as well as malate dehydrogenase (Cetica et al., 2003). The reactions mediated by these enzymes produce substrates for the TCA cycle such as pyruvate, oxaloacetate and malate. Thus, CC could potentially provide the oocyte with not only amino acids, but also a range of intermediates for oxidative metabolism (Cetica et al., 
2003). Another example of cooperativity is the facilitative action that CC exert upon the synthesis and accumulation of GSH within the oocyte (de Matos et al., 1997). This action might be mediated by the capacity of CC to produce substrates for GSH synthesis such as cysteine (de Matos et al., 1997), or by direct gap junctional transfer of GSH to the oocyte (Mori et al., 2000).

Finally, Eppig et al., (2005) reported that some paracrine signal from fully grown murine oocytes was able to up-regulate the expression of the solute carrier family 38, member 3 (SLC8A3, a sodium-coupled neutral amino transporter) in CC (Eppig et al., 2005). This transporter was expressed in CC but not in mural GC or oocytes, and showed preference for L-alanine and L-histidine. It appears, then, that oocytes can potentially increase the availability of such amino acids for gap junctional transfer (Eppig et al., 2005). Similarly, other experiments in mice have shown that key enzymes in the glycolysis (Sugiura et al., 2005) and cholesterol synthesis (Su et al., 2008) pathways are up-regulated in $\mathrm{CC}$ compared to mural GC, and that this expression pattern is controlled by oocyte derived factors. However, this phenomenon may be species-specific, as Sutton et al., (2003) did not find an oocyte-mediated effect on bovine CC carbohydrate metabolism. Such differences could also be due to different methodologies and media composition, as Zuelke and Brackett (1992) previously found increased glycolytic activity in bovine COC but not if these cumulus complexes were oocytectomised.

\section{Fatty acids as an endogenous energy source during oocyte maturation}

After the breackdown of gap junctional contact following the preovulatrory surge of gonadotrophins, the final stages of oocyte maturation as well as fertilisation and early embryo development rely on the extracellular environment and endogenous substrates for energy homeostasis. Potential endogenous energy sources are glycogen, fatty acids and proteins. Mammalian oocytes and embryos contain relatively low levels of glycogen and lipids, except for farm species, which present characteristically large quantities of intracellular lipid, triglycerides being the major component (McEvoy et al., 2000; Sturmey et al., 2009). Glycogen accumulates during embryo cleavage and it is likely to be more important at the blastocyst stage (Flynn and Hillman, 1980; Ferguson and Leese, 2006). Proteins are unlikely to be used for ATP production, moreover glycogen and protein endogenous stores are insufficient to sustain development (Ferguson and Leese, 2006; Sturmey et al., 2009) and references therein). On the other hand, lipid droplets accumulate during oocyte growth, and fatty acid oxidation can generate high number of ATP molecules (around 106 ATP molecules from the complete oxidation of one molecule of palmitate), making these molecules good candidates for energy provision during oocyte maturation and early embryo development, especially in farm animals (Sturmey et al., 2009).

A number of studies have reported the association of mitochondria with lipid inclusions, vesicles and endoplasmic reticulum during oocyte maturation (Fair et al., 1997; Motta et al., 2000; Nagano et al., 2006; Sturmey et al., 2006). The association of mitochondria and lipid droplets is suggested to serve a source of fatty acids for mitochondrial oxidation (Nagano et al., 2006; Sturmey et al., 2006). Several lines of evidence support the idea that fatty acid oxidation is a source of ATP essential for oocyte maturation and beneficial during embryo development. For example, the triglyceride content of porcine and bovine oocytes decreases during IVM, as well as after fertilisation in the bovine (Ferguson and Leese, 1999; Sturmey and Leese, 2003). Mouse embryos have been shown to take up and oxidise radiolabeled palmitate (Flynn and Hillman, 1980). In addition, lipase activity increases in bovine oocytes following IVM (Cetica et al., 2002), and the expression of the rate-limiting enzyme of $\beta$-oxidation, carnitine palmitoyl transferase 1B (Cpt1b), is up-regulated in murine COCs following human chorionic gonadotropin (hCG)-induced ovulation (Dunning et al., 2010). Inhibition of $\beta$-oxidation during oocyte IVM blocked AMP-activated protein kinase-mediated meiotic resumption in the mouse (Downs et al., 2009) and impaired oocyte developmental competence in the mouse, pig and cow (Ferguson and Leese, 2006; Sturmey et al., 2006; Dunning et al., 2010). Moreover, inhibition of $\beta$-oxidation during embryo culture negatively affects blastocyst development in the mouse and cow (Hewitson et al., 1996; Ferguson and Leese, 2006; Dunning et al., 2010). It appears that mouse and porcine embryos can, to some extent, compensate for such inhibition by adjusting glucose metabolism (Hewitson et al., 1996; Sturmey and Leese, 2008). Finally, recent experiments have shown some improvement in embryo development following supplementation of murine and porcine IVM culture media (Dunning et al., 2010; Somfai et al., 2011) and bovine embryo culture media (Sutton-McDowall et al., 2012) with L-carnitine, a cofactor of Cpt1 present in plasma. The beneficial effect of $L$-carnitine could be due to its antiapoptotic action, its antioxidant action (with a concomitant increase of intracellular GSH in oocytes), and/or the up-regulation of $\beta$-oxidation (Dunning et al., 2010; Somfai et al., 2011; Sutton-McDowall et al., 2012). It is hypothesised that up-regulation of $\beta$-oxidation might result in increased availability of carbohydrates such as glucose for its use in other non-ATP producing pathways including synthesis of nucleic acids and hyaluronic acid, cell signalling (Sutton-McDowall et al., 2012) and redox regulation. This situation may also aid metabolic regulation and rapid cell proliferation in embryos by means of the Warburg Effect (Krisher and Prather, 2012; Redel et al., 2012). Yet, the culture of oocytes/embryos without external energy substrates only supports limited development (Downs and Hudson, 2000; Ferguson and Leese, 2006; Sutton-McDowall et al., 2012), indicating that endogenous stores might not to be sufficient to fully support oocyte and embryo developmental potential, especially in those species with little lipid stores such as the mouse.

\section{Conclusion}

Like in tumours, follicular metabolism is very diverse and is aimed at energy production and branched anabolism to support growth. At first sight, tumour metabolism is reminiscent of stem cell and their inner cell mass precursors, which display high glycolytic flux and low oxphos (the so called "Warburg effect"). Therefore, metabolism of the growing oocyte and early embryo might be different to tumour metabolism. However, in vivo metabolomic analyses suggest the existence of a continuum of bioenergetic remodelling in rat tumours according to tumour size and its rate of growth with both glycolytic tumours and oxidative tumours (Jose et al., 2011). Furthermore, the Warburg effect has been shown to be mediated by an embryonic isoform of pyruvate kinase (M2 isoform, the major isoform in mammalian oocytes and early embryos (Krisher and Prather, 2012), suggesting that specificities of tumour cells 
might be operative in growing oocytes. The most striking similarity between tumour and oocyte metabolism is the importance of other, non-glucose-dependent, metabolic pathways such as fatty acid synthesis and catabolism ( $\beta$-oxidation) and glutamine catabolism (glutaminolysis) (Biswas et al., 2012; Jose et al., 2011; Daye and Wellen, 2012). The metabolic pathways essential for both oocyte and tumour growth not only supply for energy demand, but also regulate the intracellular redox potential and provide reducing equivalent used for biosynthesis of lipids, proteins and RNAs. Therefore, one should consider oocyte metabolism not only for its impact on the energetic load but also on redox potential and anabolism.

The possibility to undertake the metabolic profiling of spent culture media by single oocytes/follicles combined with live imaging of the same oocyte/follicle, should make it possible to determine the metabolome of a healthy follicle for the first time. In addition, the emerging techniques provided by "the omics" (i.e. gene expression, metabolome and fluxome profiling) might open up new possibilities for the study of oocyte/follicle and embryo metabolism; giving it a multilevel, wider and more dynamic scope. Emphasising not only the uptake of substrates, but also the intermediary metabolism, metabolic pathways and fluxes, should be key for understanding normal follicle function and its regulation.

\section{References}

ACKERT, C.L., GITTENS, J.E.I., O'BRIEN, M.J., EPPIG, J.J. and KIDDER, G.M. (2001). Intercellular communication via connexin43 gap junctions is required for ovarian folliculogenesis in the mouse. Dev Biol 233: 258-270.

AJDUK, A., MALAGOCKI, A. and MALESZEWSKI, M. (2008). Cytoplasmic maturation of mammalian oocytes: Development of a mechanism responsible for sperminduced ca2+ oscillations. Reprod Biol 8: 3-22.

BIGGERS, J.D., WHITTINGHAM, D.G. and DONAHUE, R.P. (1967). The pattern of energy metabolism in the mouse oocyte and zygote. Proc Natl Acad Sci U S A 58: 560-567.

BILODEAU-GOESEELS, S. (2006). Effects of culture media and energy sources on the inhibition of nuclear maturation in bovine oocytes. Theriogenology66:297-306.

BINELLI, M. and MURPHY, B.D. (2010). Coordinated regulation of follicle development by germ and somatic cells. Reprod Fertil Dev 22: 1-12.

BISWAS, S., LUNEC, J. and BARTLETT, K. (2012). Non-glucose metabolism in cancer cells - is it all in the fat? Cancer Metast Rev DOI: 10.1007/s10555-012-9384-6

BOLAND, N.I., HUMPHERSON, P.G., LEESE, H.J. and GOSDEN, R.G. (1994a). Characterization of follicular energy metabolism. Hum Reprod 9: 604-609.

BOLAND, N.I., HUMPHERSON, P.G., LEESE, H.J. and GOSDEN, R.G. (1994b). The effect of glucose metabolism on murine follicle development and steroidogenesis in vitro. Hum Reprod 9: 617-623.

BOOTH, P.J., HUMPHERSON, P.G., WATSON, T.J. and LEESE, H.J. (2005). Amino acid depletion and appearance during porcine preimplantation embryo development in vitro. Reproduction 130: 655-668.

BRINSTER, R.L. and HARSTAD, H. (1977). Energy metabolism in primordial germ cells of the mouse. Exp Cell Res 109: 111-117.

BURTON, G.J., JAUNIAUX, E. and CHARNOCK-JONES, D.S. (2010). The influence of the intrauterine environment on human placental development. Int $J$ Dev Biol 54: 303-312.

CARABATSOS, M.J., SELLITTO, C., GOODENOUGH, D.A. and ALBERTINI, D.F. (2000). Oocyte-granulosa cell heterologous gap junctions are required for the coordination of nuclear and cytoplasmic meiotic competence. Dev Biol226: 167-179.

CETICA, P., PINTOS, L., DALVIT, G. and BECONI, M. (2002). Activity of key enzymes involved in glucose and triglyceride catabolism during bovine oocyte maturation in vitro. Reproduction 124: 675-681.

CETICA, P., PINTOS, L., DALVIT, G. and BECONI, M. (2003). Involvement of enzymes of amino acid metabolism and tricarboxylic acid cycle in bovine oocyte maturation in vitro. Reproduction 126: 753-763.
CHAND, A.L. and LEGGE, M. (2011). Amino acid transport system I activity in developing mouse ovarian follicles. Hum Reprod 26: 3102-3108.

CLARK, A.R. and STOKES, Y.M. (2011). Follicle structure influences the availability of oxygen to the oocyte in antral follicles. Comput Math Methods Med 2011: 287186 DOI: $10.1155 / 2011 / 287186$

DAYE, D. and WELLEN, K.E. (2012). Metabolic reprogramming in cancer: Unraveling the role of glutamine in tumorigenesis. Semin Cell Dev Biol 23: 362-369.

DE MATOS, D.G., FURNUS, C.C. and MOSES, D.F. (1997). Glutathione synthesis during in vitro maturation of bovine oocytes: Role of cumulus cells. BiolReprod 57: 1420-1425.

DOWNS, S.M. (1995). The influence of glucose, cumulus cells, and metabolic coupling on atp levels and meiotic control in the isolated mouse oocyte. Dev Biol 167: 502-512.

DOWNS, S.M. and HUDSON, E.D. (2000). Energy substrates and the completion of spontaneous meiotic maturation. Zygote 8: 339-351.

DOWNS, S.M., HUMPHERSON, P.G., MARTIN, K.L. and LEESE, H.J. (1996). Glucose utilization during gonadotropin-induced meiotic maturation in cumulus cell-enclosed mouse oocytes. Mol Reprod Dev 44: 121-131.

DOWNS, S.M., HUMPHERSON, P.G. and LEESE, H.J. (1998) Meiotic induction in cumulus cell-enclosed mouse oocytes: involvement of the pentose phosphate pathway. Biol Reprod 58: 1084-1094.

DOWNS, S.M., MOSEY, J.L. and KLINGER, J. (2009). Fatty acid oxidation and meiotic resumption in mouse oocytes. Mol ReprodDev 76: 844-853.

DUMOLLARD, R., CARROLL, J., DUCHEN, M.R., CAMPBELL, K. and SWANN, K. (2009). Mitochondrial function and redox state in mammalian embryos. Semin Cell Dev Biol 20: 346-353.

DUMOLLARD, R., DUCHEN, M. and CARROLL, J. (2007). The role of mitochondrial function in the oocyte and embryo. Curr Top Dev Biol 77: 21-49.

DUMOLLARD, R., DUCHEN, M. and SARDET, C. (2006). Calcium signals and mitochondria at fertilisation. Semin Cell Dev Biol 17: 314-323.

DUNNING, K.R., CASHMAN, K., RUSSELL, D.L., THOMPSON, J.G., NORMAN, R.J and ROBKER, R.L. (2010). Beta-oxidation is essential for mouse oocyte developmental competence and early embryo development. Biol Reprod 83: 909-918.

EPPIG, J.J. (1976). Analysis of mouse oogenesis in vitro. Oocyte isolation and the utilization of exogenous energy sources by growing oocytes. J Exp Zool198: 375-382.

EPPIG, J.J. (1996). Coordination of nuclear and cytoplasmic oocyte maturation in eutherian mammals. Reprod Fertil Dev 8: 485-489.

EPPIG, J.J., PENDOLA, F.L., WIGGLESWORTH, K. and PENDOLA, J.K. (2005). Mouse oocytes regulate metabolic cooperativity between granulosa cells and oocytes: Amino acid transport. Biol Reprod 73: 351-357.

FAGBOHUN, C.F. and DOWNS, S.M. (1992). Requirement for glucose in ligandstimulated meiotic maturation of cumulus cell-enclosed mouse oocytes. J Reprod Fertil 96: 681-697.

FAIR, T., HULSHOF, S.C., HYTTEL, P., GREVE, T. and BOLAND, M. (1997). Oocyte ultrastructure in bovine primordial to early tertiary follicles. Anat Embryol (Berl) 195: 327-336.

FERGUSON, E.M. and LEESE, H.J. (1999). Triglyceride content of bovine oocytes and early embryos. J Reprod Fertil 116: 373-378.

FERGUSON, E.M. and LEESE, H.J. (2006). A potential role for triglyceride as an energy source during bovine oocyte maturation and early embryo development. Mol Reprod Dev 73: 1195-1201.

FERREIRA, E.M., VIREQUE, A.A., ADONA, P.R., MEIRELLES, F.V., FERRIANI, R.A. and NAVARRO, P.A. (2009). Cytoplasmic maturation of bovine oocytes: Structura and biochemical modifications and acquisition of developmental competence. Theriogenology 71 : 836-848.

FISCHER, B. and BAVISTER, B.D. (1993). Oxygen tension in the oviduct and uterus of rhesus monkeys, hamsters and rabbits. J Reprod Fertil 99: 673-679.

FLYNN, T.J. and HILLMAN, N. (1980). The metabolism of exogenous fatty acids by preimplantation mouse embryos developing in vitro. J Embryol Exp Morphol 56: 157-168.

GARDNER, D.K., WALE, P.L., COLLINS, R. and LANE, M. (2011). Glucose consumption of single post-compaction human embryos is predictive of embryo sex and live birth outcome. Hum Reprod 26: 1981-1986.

GRIFFIN, J., EMERY, B.R., HUANG, I., PETERSON, C.M. and CARRELL, D.T. (2006). Comparative analysis of follicle morphology and oocyte diameter in four 
mammalian species (mouse, hamster, pig, and human). J Exp Clin Assist Reprod 3: 2 DOI: 10.1186/1743-1050-3-2

HARRIS, S.E., ADRIAENS, I., LEESE, H.J., GOSDEN, R.G. and PICTON, H.M. (2007). Carbohydrate metabolism by murine ovarian follicles and oocytes grown in vitro. Reproduction 134: 415-424.

HARRIS, S.E., GOPICHANDRAN, N., PICTON, H.M., LEESE, H.J. and ORSI, N. (2005). Nutrient concentrations in murine follicular fluid and the female reproductive tract. Theriogenology 64: 992-1006.

HARRIS, S.E., LEESE, H.J., GOSDEN, R.G. and PICTON, H.M. (2009). Pyruvate and oxygen consumption throughout the growth and development of murine oocytes. Molecular Reprod Dev 76: 231-238

HEMMINGS, K.E., LEESE, H.J. and PICTON, H.M. (2012). Amino acid turnover by bovine oocytes provides an index of oocyte developmental competence in vitro. Biol Reprod 86: 165, 161-112.

HONG, J. and LEE, E. (2007). Intrafollicular amino acid concentration and the effect of amino acids in a defined maturation medium on porcine oocyte maturation, fertilization, and preimplantation development. Theriogenology 68: 728-735.

HOUGHTON, F.D., HAWKHEAD, J.A., HUMPHERSON, P.G., HOGG, J.E., BALEN A.H., RUTHERFORD, A.J. and LEESE, H.J. (2003). Non-invasive amino acid turnover predicts human embryo developmental capacity. Hum Reprod 18: 1756-1757.

HUGENTOBLER, S.A., DISKIN, M.G., LEESE, H.J., HUMPHERSON, P.G., WATSON, T., SREENAN, J.M. and MORRIS, D.G. (2007). Amino acids in oviduct and uterine fluid and blood plasma during the estrous cycle in the bovine. $\mathrm{Mo}$ Reprod Dev 74: 445-454.

ISHIZAKI, C., WATANABE, H., BHUIYAN, M.M.U. and FUKUI, Y. (2009) Developmental competence of porcine oocytes selected by brilliant cresyl blue and matured individually in a chemically defined culture medium. Theriogenology 72: 72-80.

JANSEN, R.P. and DE BOER, K. (1998). The bottleneck: Mitochondrial imperatives in oogenesis and ovarian follicular fate. Mol Cell Endocrinol 145: 81-88.

JOHNSON, M.T., FREEMAN, E.A., GARDNER, D.K. and HUNT, P.A. (2007). Oxidative metabolism of pyruvate is required for meiotic maturation of murine oocytes in vivo. Biol Reprod 77: 2-8.

JOHNSON, M.T., MAHMOOD, S. and PATEL, M.S. (2003). Intermediary metabolism and energetics during murine early embryogenesis. JBiol Chem278:31457-31460.

JOSE, C., BELLANCE, N. and ROSSIGNOL, R. (2011). Choosing between glycolysis and oxidative phosphorylation: A tumor's dilemma? Biochi Biophys Acta - Bioenergetics 1807: 552-561.

KOL, S., BEN-SHLOMO, I., RUUTIAINEN, K., ANDO, M., DAVIES-HILL, T.M., ROHAN, R.M., SIMPSON, I.A. and ADASHI, E.Y. (1997). The midcycle increase in ovarian glucose uptake is associated with enhanced expression of glucose transporter 3. Possible role for interleukin-1, a putative intermediary in the ovulatory process. J Clin Invest 99: 2274-2283.

KRISHER, R.L., BRAD, A.M., HERRICK, J.R., SPARMAN, M.L. and SWAIN, J.E. (2007). A comparative analysis of metabolism and viability in porcine oocytes during in vitro maturation. Anim Reprod Sci 98: 72-96

KRISHER, R.L. and PRATHER, R.S. (2012). A role for the warburg effect in preimplantation embryo development: Metabolic modification to support rapid cell proliferation. Mol Reprod and Dev 79: 311-320.

LEESE, H.J. (2012). Metabolism of the preimplantation embryo: 40 years on. $R e$ production 143: 417-427.

LEESE, H.J., BAUMANN, C.G., BRISON, D.R., MCEVOY, T.G. and STURMEY, R.G. (2008). Metabolism of the viable mammalian embryo: Quietness revisited. $\mathrm{Mol}$ Hum Reprod 14: 667-672.

LUBERDA, Z. (2005). The role of glutathione in mammalian gametes. Reprod Biol 5: $5-17$

MCEVOY, T., COULL, G., BROADBENT, P., HUTCHINSON, J. and SPEAKE, B. (2000). Fatty acid composition of lipids in immature cattle, pig and sheep oocytes with intact zona pellucida. J Reprod Fertil 118: 163-170

MCLAUGHLIN, E.A. and MCIVER, S.C. (2009). Awakening the oocyte: Controlling primordial follicle development. Reproduction 137: 1-11.

MITCHELL, P.A. and BURGHARDT, R.C. (1986). The ontogeny of nexuses (gap junctions) in the ovary of the fetal mouse. Anat Rec 214: 283-288.

MORI, T., AMANO, T. and SHIMIZU, H. (2000). Roles of gap junctional communication of cumulus cells in cytoplasmic maturation of porcine oocytes cultured in vitro. Biol Reprod 62: 913-919.
MOTTA, P.M., MAKABE, S. and NOTTOLA, S.A. (1997). The ultrastructure of human reproduction. I. The natural history of the female germ cell: Origin, migration and differentiation inside the developing ovary. Hum Reprod Update 3: 281-295.

MOTTA, P.M., NOTTOLA, S.A., MAKABE, S. and HEYN, R. (2000). Mitochondrial morphology in human fetal and adult female germ cells. Hum Reprod 15 Suppl 2: $129-147$.

NAGANO, M., KATAGIRI, S. and TAKAHASHI, Y. (2006). Atp content and maturational/ developmental ability of bovine oocytes with various cytoplasmic morphologies. Zygote 14: 299-304.

PARTRIDGE, R.J. and LEESE, H.J. (1996). Consumption of amino acids by bovine preimplantation embryos. Reprod Fertil Dev 8: 945-950.

PELLAND, A.M.D., CORBETT, H.E. and BALTZ, J.M. (2009). Amino acid transport mechanisms in mouse oocytes during growth and meiotic maturation. Biol. Reprod. 81: 1041-1054.

PICTON, H., BRIGGS, D. and GOSDEN, R. (1998). The molecular basis of oocyte growth and development. Mol Cell Endocrinol 145: 27-37.

PICTON, H.M. (2001). Activation of follicle development: The primordial follicle. Theriogenology 55: 1193-1210.

PICTON, H.M., ELDER, K., HOUGHTON, F.D., HAWKHEAD, J.A., RUTHERFORD A.J., HOGG, J.E., LEESE, H.J. and HARRIS, S.E. (2010). Association between amino acid turnover and chromosome aneuploidy during human preimplantation embryo development in vitro. Mol Hum Reprod 16: 557-569.

PURCELL, S.H. and MOLEY, K.H. (2009). Glucose transporters in gametes and preimplantation embryos. Trends Endocrinol Metab 20: 483-489.

REDDING, G.P., BRONLUND, J.E. and HART, A.L. (2007). Mathematical modelling of oxygen transport-limited follicle growth. Reproduction 133: 1095-1106.

REDDING, G.P., BRONLUND, J.E. and HART, A.L. (2008). Theoretical investigation into the dissolved oxygen levels in follicular fluid of the developing human follicle using mathematical modelling. Reprod Fertil Dev 20: 408-417.

REDEL, B.K., BROWN, A.N., SPATE, L.D., WHITWORTH, K.M., GREEN, J.A. and PRATHER, R.S. (2012). Glycolysis in preimplantation development is partially controlled by the warburg effect. Mol ReprodDev 79: 262-271.

RIEGER, D. and LOSKUTOFF, N.M. (1994). Changes in the metabolism of glucose, pyruvate, glutamine and glycine during maturation of cattle oocytes in vitro. $J$ Reprod Fertil 100: 257-262.

ROBERTS, R., STARK, J., IATROPOULOU, A., BECKER, D.L., FRANKS, S. and HARDY, K. (2004). Energy substrate metabolism of mouse cumulus-oocyte complexes: Response to follicle-stimulating hormone is mediated by the phosphatidylinositol 3-kinase pathway and is associated with oocyte maturation. Bio Reprod 71: 199-209.

ROY, S.K. and TERADA, D.M. (1999). Activities of glucose metabolic enzymes in human preantral follicles: In vitro modulation by follicle-stimulating hormone luteinizing hormone, epidermal growth factor, insulin-like growth factor $\mathrm{i}$, and transforming growth factor $\beta 1$. Biol Reprod 60: 763-768.

SCHULTZ, R.M., LETOURNEAU, G.E. and WASSARMAN, P.M. (1979). Program of early development in the mammal: Changes in the patterns and absolute rates of tubulin and total protein synthesis during oocyte growth in the mouse. Dev Biol 73: 120-133.

SOMFAI, T., KANEDA, M., AKAGI, S., WATANABE, S., HARAGUCHI, S., MIZUTANI, E., DANG-NGUYEN, T.Q., GESHI, M., KIKUCHI, K. and NAGAI, T. (2011). Enhancement of lipid metabolism with I-carnitine during in vitro maturation improves nuclear maturation and cleavage ability of follicular porcine oocytes. Reprod Fertil Dev 23: 912-920.

STERNLICHT, A.L. and SCHULTZ, R.M. (1981). Biochemical studies of mammalian oogenesis: Kinetics of accumulation of total and poly(a)-containing rna during growth of the mouse oocyte. J Exp Zool 215: 191-200.

STURMEY, R.G., BRISON, D.R. and LEESE, H.J. (2008). Symposium: Innovative techniques in human embryo viability assessment. Assessing embryo viability by measurement of amino acid turnover. Reprod Biomed Online 17: 486-496.

STURMEY, R.G. and LEESE, H.J. (2003). Energy metabolism in pig oocytes and early embryos. Reproduction 126: 197-204.

STURMEY, R.G. and LEESE, H.J. (2008). Role of glucose and fatty acid metabolism in porcine early embryo development. ReprodFertil Dev 20 pp. 149 (Abstr.)

STURMEY, R.G., O'TOOLE, P.J. and LEESE, H.J. (2006). Fluorescence resonance energy transfer analysis of mitochondrial:Lipid association in the porcine oocyte. Reproduction 132: 829-837. 
STURMEY, R.G., REIS, A., LEESE, H.J. and MCEVOY, T.G. (2009). Role of fatty acids in energy provision during oocyte maturation and early embryo development. Reprod Domest Anim 44 Suppl 3: 50-58.

SU, J., WANG, Y., LI, R., PENG, H., HUA, S., LI, Q., QUAN, F., GUO, Z. and ZHANG, Y. (2012) Oocytes selected using BCB staining enhance nuclear reprogramming and the in vivo development of SCNT embryos in cattle. PLoS One 7, e36181.

SU, Y.Q., SUGIURA, K. and EPPIG, J.J. (2009). Mouse oocyte control of granulosa cell development and function: Paracrine regulation of cumulus cell metabolism. Semin Reprod Med 27: 032-042.

SU, Y.Q., SUGIURA, K., WIGGLESWORTH, K., O'BRIEN, M.J., AFFOURTIT, J.P., PANGAS, S.A., MATZUK, M.M. and EPPIG, J.J. (2008). Oocyte regulation of metabolic cooperativity between mouse cumulus cells and oocytes: Bmp15 and gdf9 control cholesterol biosynthesis in cumulus cells. Development 135: 111-121.

SUGIURA, K., PENDOLA, F.L. and EPPIG, J.J. (2005). Oocyte control of metabolic cooperativity between oocytes and companion granulosa cells: Energy metabolism. Dev Biol 279: 20-30.

SUTTON-MCDOWALL, M.L., FEIL, D., ROBKER, R.L., THOMPSON, J.G. and DUNNING, K.R. (2012). Utilization of endogenous fatty acid stores for energy production in bovine preimplantation embryos. Theriogenology 77: 1632-1641.

SUTTON-MCDOWALL, M.L., GILCHRIST, R.B. and THOMPSON, J.G. (2004). Cumulus expansion and glucose utilisation by bovine cumulus-oocyte complexes during in vitro maturation: The influence of glucosamine and follicle-stimulating hormone. Reproduction 128: 313-319.

SUTTON-MCDOWALL, M.L., GILCHRIST, R.B. and THOMPSON, J.G. (2010). The pivotal role of glucose metabolism in determining oocyte developmental competence. Reproduction 139: 685-695.

SUTTON, M.L., CETICA, P.D., BECONI, M.T., KIND, K.L., GILCHRIST, R.B. and THOMPSON, J.G. (2003a). Influence of oocyte-secreted factors and culture duration on the metabolic activity of bovine cumulus cell complexes. Reproduction 126: 27-34.

SUTTON, M.L., GILCHRIST, R.B. and THOMPSON, J.G. (2003b). Effects of in-vivo and in-vitro environments on the metabolism of the cumulus-oocyte complex and its influence on oocyte developmental capacity. Hum Reprod Update 9: 35-48.

SWAIN, J.E., BORMANN, C.L., CLARK, S.G., WALTERS, E.M., WHEELER, M.B. and KRISHER, R.L. (2002). Use of energy substrates by various stage preimplantation pig embryos produced in vivo and in vitro. Reproduction 123: 253-260.

TANGHE, S., VAN SOOM, A., NAUWYNCK, H., CORYN, M. and DE KRUIF, A.
(2002). Minireview: Functions of the cumulus oophorus during oocyte maturation, ovulation, and fertilization. Mo Reprod Dev 61: 414-424.

TOMEK, W., TORNER, H. and KANITZ, W. (2002). Comparative analysis of protein synthesis, transcription and cytoplasmic polyadenylation of mrna during maturation of bovine oocytes in vitro. Reprod Domest Anim 37: 86-91.

TRIPATHI, A., KUMAR, K.V.P. and CHAUBE, S.K. (2010). Meiotic cell cycle arrest in mammalian oocytes. J Cell Physiol 223: 592-600.

TROUNSON, A., ANDERIESZ, C. and JONES, G. (2001). Maturation of human oocytes in vitro and their developmental competence. Reproduction 121: 51-75.

VAN BLERKOM, J. (2000). Intrafollicular influences on human oocyte developmental competence: Perifollicular vascularity, oocyte metabolism and mitochondrial function. Hum Reprod 15: 173-188.

VAN BLERKOM, J. (2011). Mitochondrial function in the human oocyte and embryo and their role in developmental competence. Mitochondrion 11: 797-813.

VAN WINKLE, L. J. 2001. Amino acid transport regulation and early embryo development. Biol Reprod 64: 1-12.

WANG, Q., CHI, M.M., SCHEDL, T. and MOLEY, K.H. (2012). An intercellular pathway for glucose transport into mouse oocytes. Am J Physiol Endocrinol Metab 302: E1511-E1518.

WATSON, A.J., DE SOUSA, P., CAVENEY, A., BARCROFT, L.C., NATALE, D., URQUHART, J. and WESTHUSIN, M.E. (2000). Impact of bovine oocyte maturation media on oocyte transcript levels, blastocyst development, cell number, and apoptosis. Biol Reprod 62: 355-364.

YOUNG, J.M. and MCNEILLY, A.S. (2010). Theca: The forgotten cell of the ovarian follicle. Reproduction 140: 489-504.

ZHANG, L., JIANG, S., WOZNIAK, P.J., YANG, X. and GODKE, R.A. (1995). Cumulus cell function during bovine oocyte maturation, fertilization, and embryo development in vitro. Mol Reprod Dev 40: 338-344.

ZHENG, P. (2007). Effects of in vitro maturation of monkey oocytes on their developmental capacity. Anim Reprod Sci 98: 56-71.

ZUELKE, K.A. and BRACKETT, B.G. (1992). Effects of luteinizing hormone on glucose metabolism in cumulus-enclosed bovine oocytes matured in vitro. Endocrinology 131: $2690-2696$

ZUELKE, K.A. and BRACKETT, B.G. (1993). Increased glutamine metabolism in bovine cumulus cell-enclosed and denuded oocytes after in vitro maturation with luteinizing hormone. Biol Reprod 48: 815-820. 


\section{Further Related Reading, published previously in the Int. J. Dev. Biol.}

Hypoxia and neural stem cells: from invertebrates to brain cancer stem cells

Ferdinando Mannello, Virginia Medda and Gaetana A Tonti

Int. J. Dev. Biol. (2011) 55: 569 - 581

Modulation of mitochondrial biogenesis and bioenergetic metabolism upon in vitro and in vivodifferentiation of human ES and iPS cells

Alessandro Prigione and James Adjaye

Int. J. Dev. Biol. (2010) 54: 1729 - 1741

Intraovarian transplantation of stage I-Il follicles results in viable zebrafish embryos Zsolt Csenki, Andreas Zaucker, Balázs Kovács, Yavor Hadzhiev, Árpád Hegyi

Int. J. Dev. Biol. (2010) 54: 585 - 589

Placental metabolic reprogramming: do changes in the mix of energy-generating substrates modulate fetal growth?

Nicholas P. Illsley, Isabella Caniggia and Stacy Zamudio

Int. J. Dev. Biol. (2010) 54: 409 - 419

Large-scale production of growing oocytes in vitro from neonatal mouse ovaries Arata Honda, Michiko Hirose, Kimiko Inoue, Hitoshi Hiura, Hiromi Miki, Narumi Ogonuki, Michihiko Sugimoto, Kuniya Abe, Mito Kanatsu-Shinohara, Tomohiro Kono, Takashi Shinohara and Atsuo Ogura*

Int. J. Dev. Biol. (2009) 53: 605 - 613

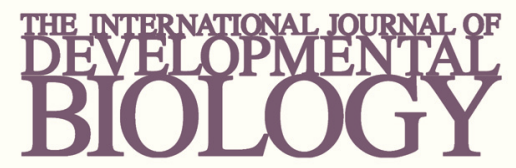

Volume 54 Nos. 6/7

Special Issue
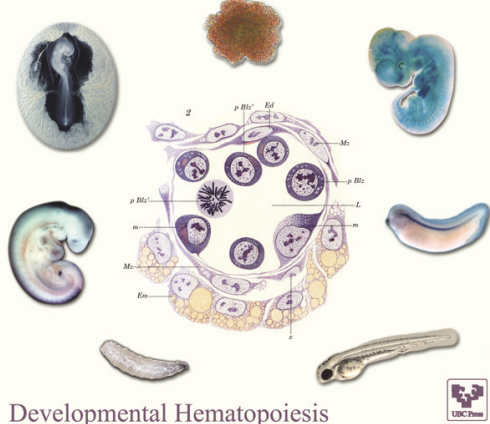

wack
5 yr ISI Impact Factor $(2011)=2.959$

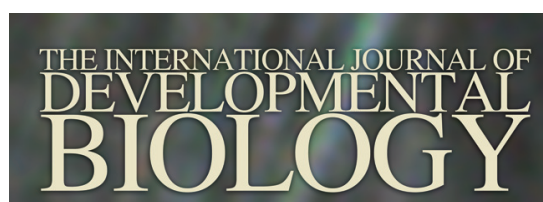

Special Issue

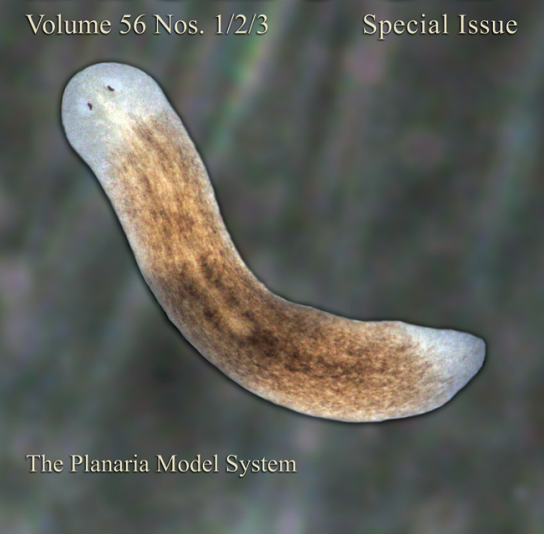

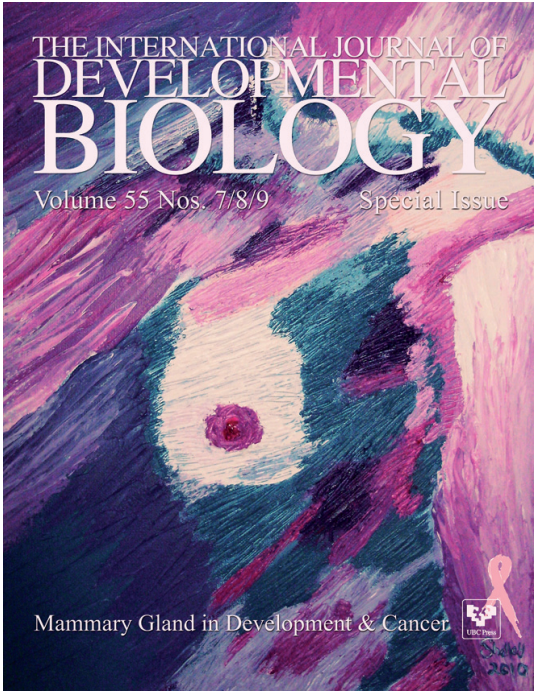

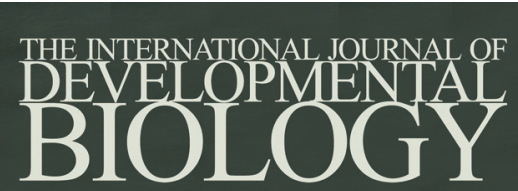

Volume 55 Nos. $4 / 5$

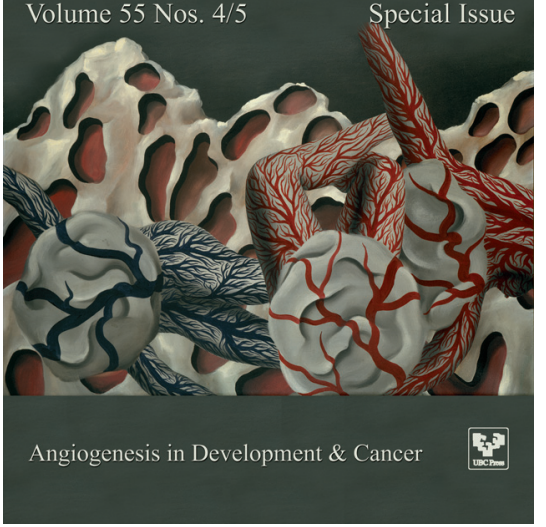

